\title{
Fetal Aortic-cerebral Doppler Resistance Index Ratio: An Indicator of Physiologic Blood Flow Distribution
}

\author{
János Aranyosi, Tamás Deli, Péter Bettembuk, Bence Kozma, Tamás Kovács, Olga Török, \\ Zoltán Tóth
}

Department of Obstetrics and Gynecology, Medical and Health Science Center, University of Debrecen, Debrecen, Hungary

Correspondence: János Aranyosi, Department of Obstetrics and Gynecology, Medical and Health Science Center, University of Debrecen Nagyerdei Krt 98, POB 37, H-4012 Debrecen, Hungary, Phone/Fax: +36-52-417171, e-mail: aranyosij@yahoo.com

\begin{abstract}
Identical hemodynamic impedance and constant ratio of the fetal descending aorta and middle cerebral artery of uncomplicated pregnancies at term.

Fetal aortic-cerebral Doppler resistance index ratio: An indicator of physiologic blood flow distribution.

Objective: To interpret the physiologic fetal arterial blood flow distribution by relating the vascular impedance of the fetal descending aorta (DA) and middle cerebral artery (MCA) and to establish the reference ranges for the aortic-cerebral Doppler resistance index ratio $(\mathrm{ACRI})$.

Study design: Ninety-six patients with uncomplicated pregnancies were recruited for the cross-sectional assessment of the Doppler resistance index $(\mathrm{RI})$ in the fetal DA and MCA between the 38rd and 40th weeks of gestation. The normal ranges of the ACRI were calculated. A cut-off value was designed to facilitate the clinical application of the ACRI.

Results: Between the 38th and 40th weeks of gestation in normal pregnancies the ACRI of healthy fetuses is constant, the overall mean is: $1.062(+/-0.087)$. A single cut-off value of 1.2 is recommended to assist separating normal and pathologic arterial blood flow patterns.

Conclusion: The normal ACRI reflects the identical vascular resistance of the descending aorta and the cerebral vessels, which maintains the physiologic fetal central arterial blood flow. Additional clinical studies are necessary to assess the diagnostic efficacy of the abnormal ACRI (>1.2) as a potentially useful marker of the centralized arterial circulation indicating the early stage of fetal hypoxemic jeopardy.
\end{abstract}

Keywords: Fetal blood flow, descending aorta, middle cerebral artery, Doppler index ratio.

Uteroplacental dysfunction may generate severe fetal and neonatal complications. ${ }^{1}$ Doppler ultrasound is used in obstetrics to discover the initial stage of fetal hemodynamic pathology in order to improve perinatal outcome. ${ }^{2}$ Impaired placental performance results in redistribution of the fetal arterial circulation beginning with minor changes in the vascular resistance of the DA and the MCA. The pathophysiology of the arterial redistribution has been well explained. ${ }^{3}$ Fetal hypoxia results in a decrease in the vascular resistance in the MCA, while resistance in the DA increases in correlation with the peripheral vasoconstriction in the splanchnic and skeletal region. ${ }^{3,4}$ Comparing the impedance to blood flow in different vascular areas may improve early recognition of fetal cardiovascular compensation. ${ }^{5}$ Doppler index ratios are heart rate independent and more sensitive than autonomous evaluation of the vessels, since they are expressing opposite trends within the normal ranges. ${ }^{6}$
At present there are no guidelines for the clinical definition of the physiologic fetal arterial blood flow distribution. Publications related to the ratio of blood flow in the DA and in the cerebral vessels have attempted to provide better estimation of the fetal compromise as well as the degree of oxygenation especially among small for date fetuses. ${ }^{7,17}$ To recognize circulatory readjustment normal ranges for the Doppler index ratios, reflecting the physiologic blood distribution, must be established. Given that the increased impedance in the DA is a consequence of the peripheral vasoconstriction and the resistance to blood flow in the MCA decreases due to hypoxia, we compared the hemodynamic resistance of these two vessels. To the best of our knowledge the ratio between the Pourcelot RI values obtained form the DA and the MCA in uncomplicated pregnancies has not been discussed. ${ }^{8,9}$

The aim of our study was to construct the reference limits for the ACRI between the 38th and 40th weeks of 
normal gestation and to present a cut-off value below which the arterial blood distribution is considered to be physiologic.

\section{METHODS}

Ninety-six healthy pregnant women participated voluntarily in this cross-sectional study. The research protocol was approved by the Regional Committee for Medical Research Ethics; written informed consent was obtained from all participants.

As part of the pregnancy care protocol at our university hospital, Doppler ulrasound measurements were carried out in uncomplicated pregnancies at term, and the measurements presented in this study were recorded within 24 hours before delivery. The gestational age was estimated from the last normal menstrual period and confirmed by the first or second trimester ultrasound reports (crown-rump length, biparietal diameter records). All patients delivered in our university hospital.

All examinations were performed with the same equipment (ATL HDI-3000, Advanced Technology Laboratories, Bothen Washington) by a single operator. The technique of imaging and Doppler velocimetry was similar to the previously described methods. ${ }^{9,10}$ A 3.5-5 MHz curvilinear transducer recorded the pulsed wave ultrasound signals. The spatial peak temporal average intensity was always less then $94 \mathrm{~mW} / \mathrm{cm}^{2}$. The high pass filter was set at the minimum of $50 \mathrm{~Hz}$. The size of the sampling volume ranged between 1.5-6 $\mathrm{mm}$ to cover the vascular diameter entirely. The insonation angle remained below 50 degrees in all cases.
Recordings were done during fetal apnea and inactivity, and the heart rate ranged from 120 to 160 beats per minute. The patients were lying in a semirecumbent position with slightly elevated chest and head. Blood flow measurements were performed after finding the target vessels by real time scan assisted by the color flow imaging. Special care was taken to chose standard sampling points along the blood vessels. $^{23}$ The Doppler velocity waveforms were obtained from the abdominal aorta below the origin of the renal arteries (Fig. 1). The MCA was imaged in the transverse view of the fetal head at the level of the biparietal diameter, as the major anterolateral branch of the circle of Willis is in proximity to the greater wing of the sphenoid (Fig. 2). Samples were measured on the distal segment of the MCA. Compression of the fetal head by the transducer was avoided. The Doppler sample was directed into the lumen of the vessels to receive the maximal audible signal and frequency shift. Analysis of waveforms was performed using the Doppler shift spectrum. RI was calculated from at least 8 to 16 satisfactory waveforms by the factory provided software of the equipment.

The ACRI values were calculated from the RI of the DA and MCA. The reference limits of ACRI (means and standard deviations) were set up on the basis of 96 examinations. Data analysis was performed with SPSS software (version 7.0). Normality was checked for each variable. Analysis of variance was used to prove that the ACRI is even between the 38th and the 40th weeks. In order to separate the normal and abnormal ACRI results the upper limit of the normal range was determined by calculating 2 standard deviations over the highest (38 weeks) mean value.

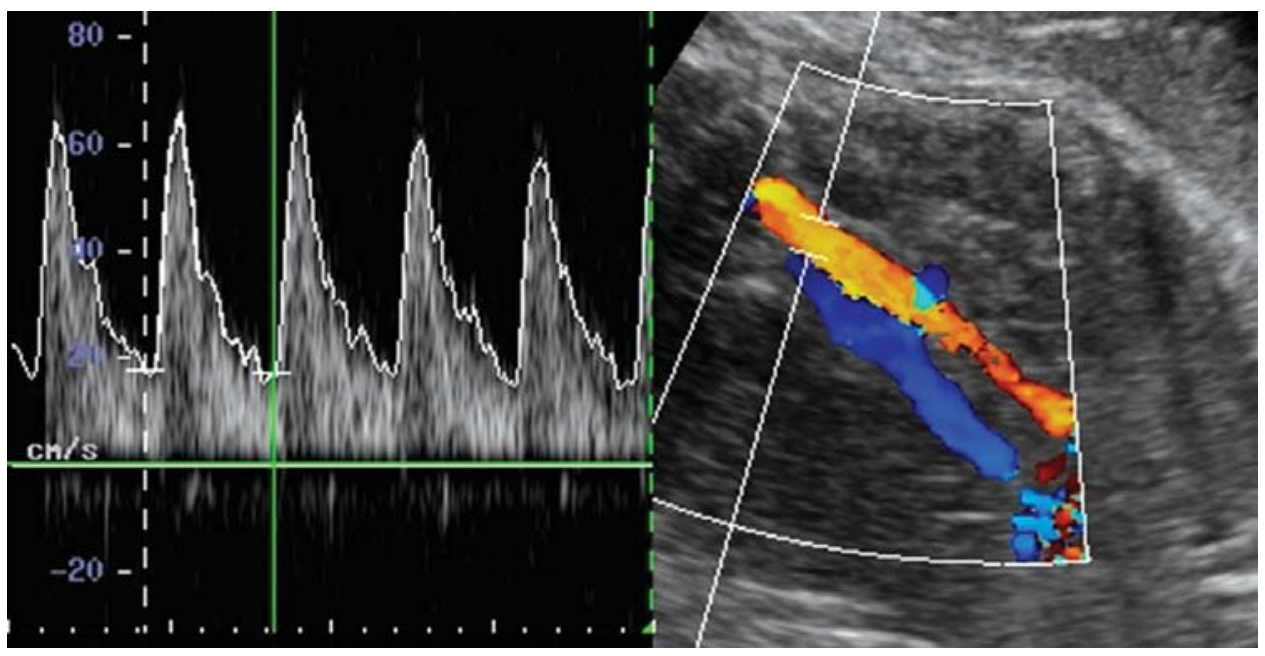

Fig. 1: Normal Doppler velocity waveforms obtained from the abdominal aorta below the origin of the renal arteries 


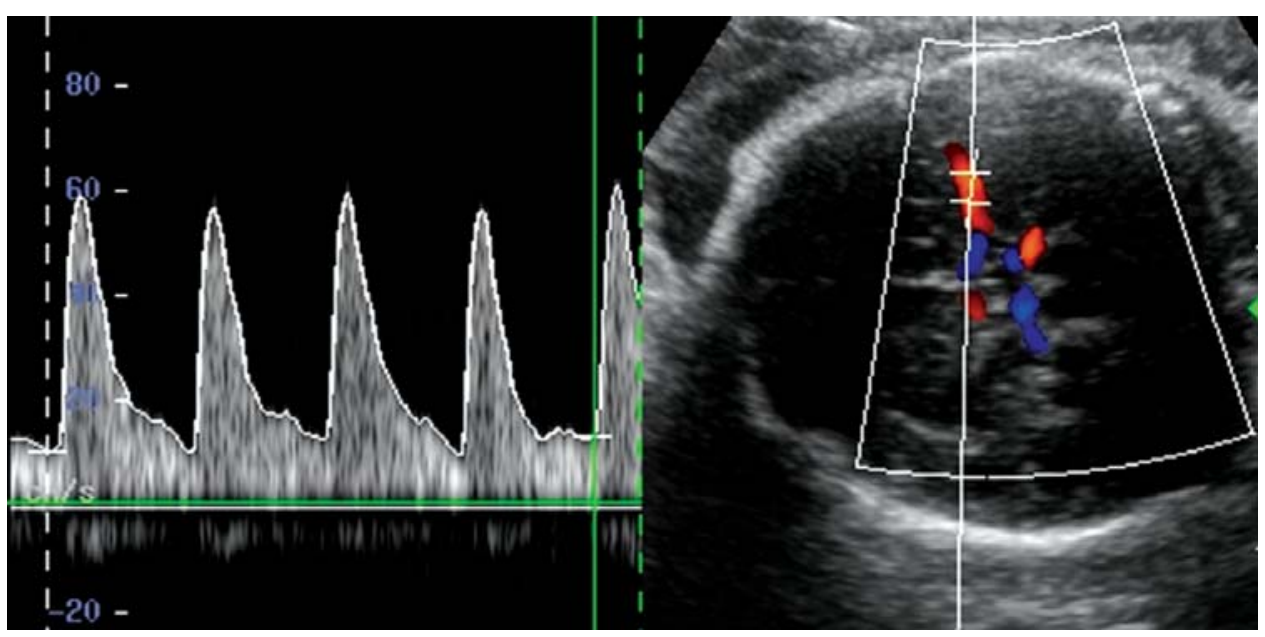

Fig. 2: Normal Doppler velocity waveforms obtained from the middle cerebral arteries

\section{RESULTS}

In the study population the mean duration of gestation was $38.8(+/-0.8)$ weeks, the median birth weight of the newborns was $3484(+/-523)$ grams and the 1-minute Apgar score was 9.81 in average. Baseline characteristics of the study population are presented in Table 1.

The weekly mean values of the ACRI and the standard deviations between the 38th and the 40th weeks of gestation with the number of Doppler examinations are presented in Table 2. The mean values of the ACRI are close to 1.0. The analysis of variance proved no statistical difference between the weekly mean values, therefore the ACRI can be considered constant between the 38th and 40th weeks of pregnancy with an overall mean of $1.062(+/-0.087)$.

The value of the cut-off limit (overall mean $+2 \mathrm{SD}$ ) to help classifying the normal and abnormal ACRI results equals

Table 1: Baseline characteristics of the study population

$\begin{array}{ll}\text { Number of patients } & 96 \\ \text { Maternal age: mean year (range) } & 28(18-43) \\ \text { Gestational age in weeks: mean (SD) } & 38.8(0.8) \\ \text { Birth weight: gramms mean (SD) } & 3484(523) \\ \text { One minute Apgar score: mean } & 9.81\end{array}$

Table 2: The weekly mean values and standard deviations (SD) of the fetal aortic to cerebral resistance Index ratio (ACRI) between 38 and 40 weeks of gestation are presented with the number of Doppler examinations

$\begin{array}{cccc}\begin{array}{c}\text { Gestational } \\ \text { age (weeks) }\end{array} & \begin{array}{c}\text { Number of } \\ \text { examinations }\end{array} & \begin{array}{c}\text { ACRI } \\ \text { mean }\end{array} & S D \\ 38 & 34 & 1.0782 & 0.0833 \\ 39 & 38 & 1.0534 & 0.1021 \\ 40 & 24 & 1.0535 & 0.0593\end{array}$

with 1.236. To facilitate the practical application of the ACRI, the results were considered normal below the level of 1.2.

\section{DISCUSSION}

In this paper we present our observation that the Resistance Indices of the DA and MCA of healthy fetuses are identical reflecting the physiologic arterial blood flow distribution.

The recognition of the initial hemodynamic changes in the fetal arterial circulation is based on the knowledge of the physiologic blood flow supply and the reference limits of the Doppler indices. Several authors have introduced normal Doppler values for the umbilical artery (UA), DA and MCA. ${ }^{9-11,18-20}$ Recently, the relation between the impedance of different vascular areas, including mainly the umbilical and cerebral arteries, has been studied. 5,12,21,22 Doppler index ratios are more accurate than the individual examination of the vessels, because they emphasize the opposite trend of the preliminary alterations of the vascular resistance in different fetal arteries. Previous papers found that the umbilical-cerebral ratio identifies differential blood flow between the placenta and the fetal brain. ${ }^{13}$ The abnormal umbilical-cerebral ratio is associated with high incidence of intrauterine growth retardation (IUGR), adverse perinatal outcome, and also correlates with the changes of fetal oxygenation. ${ }^{6,12-14}$ The ratio of aortic mean velocity and the common carotid artery pulsatility index was a strong predictor of asphyxia for IUGR fetuses. ${ }^{7,17}$ However, none of the papers described a Doppler index ratio, which reflects the balance of the fetal arterial blood distribution under physiologic circumstances.

Increased resistance in the umbilical artery is not necessarily correlated with impaired placental performance. 
Moreover, placental insufficiency as well as fetal hypoxia may develop in case of normal umbilical artery blood flow. ${ }^{15,16}$ The purpose of the obstetrical Doppler measurement is the proper assessment of the actual fetal condition. Therefore, we intended to apply a Doppler index ratio, which illustrates the central arterial blood distribution independently from the placental vascular resistance. A normal aorticcerebral RI ratio (ACRI) represents the physiologic stability of the peripheral and cerebral arterial circulation.

The results of the present study indicate that the RI values of the DA and the MCA are almost identical in healthy fetuses at term between the 38th and 40th weeks, regardless of the gestational age. Consequently, the normal value of ACRI is close to 1.0 , which is nearly constant in healthy fetuses at the term of gestation. It is worth remarking here that in the case of more advanced pregnancies, we also observed a slight decrease in the RI of the MCA accompanied by an unchanged RI of the DA, and thus an increase of the ACRI (at week 41, ACRI \pm SD was found to be $1.13 \pm$ $0.11, \mathrm{n}=8$ ), in line with what has been described before by other authors as well. ${ }^{19}$ (These preliminary observations of ours require further examination due to the low number of cases so far).

As a response to hypoxia, the fetal cardiac output and blood flow is readjusted. ${ }^{2,3}$ Elevated ACRI levels represent increasing peripheral and decreasing cerebral vascular resistance at the same time, and may become apparent before the RI values of the DA and MCA reach the abnormal range.

We selected a cut-off limit to facilitate the use of ACRI, as no previous references were available. Since the ACRI is even, we calculated one single cut-off limit, which can be applied consistently between the 38th and 40th weeks of pregnancy to separate the physiologic and the pathologic fetal blood flow patterns. ACRI values below this limit represent the identical impedance of the peripheral and cerebral vessels, whereas a higher ACRI reflects the redistribution of the arterial circulation.

The assessment of fetal condition is more adequate if the velocimetry of the umbilical artery is completed by the Doppler examination of the DA and MCA. The efficacy of the method can be further improved by applying the Doppler index ratios. Our primary finding suggests that the identical hemodynamic resistance of the abdominal aorta and the cerebral arteries maintain the physiologic fetal arterial blood distribution. It is reflected by the normal ACRI values, which are nearly constant at term in uncomplicated pregnancies.
Therefore, the ACRI is not influenced by the gestational age. It is likely to be independent of the placental perfusion since the blood flow velocity waveforms in the DA are governed by chemoreceptor activity and Doppler index ratios were shown to be fetal heart-rate independent.,

The introduction of the ACRI offers a novel approach to the interpretation of the fetal arterial blood flow distribution, even before reverse or absent end-diastolic flow in the UA and/or the DA indicate a more advanced stage of fetal hypoxia. ${ }^{23}$ Although the simplistic explanation of Doppler indices should be avoided, the abnormal ACRI may be considered as a potentially useful marker of impending fetal hypoxic compromise. The results of our study are preliminary and further prospective, randomized clinical trials are necessary to assess the diagnostic value and the clinical benefits of the ACRI measurement.

\section{ACKNOWLEDGMENTS}

We are grateful to Thomas D Kerenyi MD and to Richard L Berkowitz MD (Department of Obstetrics and Gynecology, Mount Sinai School of Medicine, New York, USA) for the valuable comments.

\section{REFERENCES}

1. Barker DJP, Gluckman PD, Godfrey KM, et al. Fetal nutrition and cardiovascular disease in adult life. Lancet 1993;341:93841.

2. Neilson JP, Alfirevic Z. Doppler ultrasound for fetal assessment in high risk pregnancies. Cochrane Database Syst Rev 2000;(2):CD000073.

3. Peeters LL, Sheldon RE, Jones Jr MD, Makowski EL, Meschia G. Blood flow to fetal organs as a function of arterial oxygen content. Am J Obstet Gynecol 1979;135:637-46.

4. Hanson, MA. Do we now understand the control of fetal circulation? Eur J Obstet Gynecol Reprod Biol 1997;75:55-61.

5. Scherjon SA, Smolders-DeHaas H, Kok JH, Zondervan HA. The "brain-sparing” effect: Antenatal cerebral Doppler findings in relation to neurologic outcome in very preterm infants. Am J Obstet Gynecol 1993;169:169-75.

6. Gramellini D, Folli MC, Raboni S, Vadora E, Merialdi A. Cerebral-Umbilical Doppler Ratio As a Predictor of Adverse Perinatal Outcome. Obstet Gynecol 1992;79:416-20.

7. Arbeille P. Fetal arterial Doppler-IUGR and hypoxia. Eur J Obstet Gynecol Reprod Biol 1997;75:51-53.

8. Bilardo CM, Nicolaides KH, Campbell S. Doppler measurement of fetal and uteroplacental circulations: Relationship with umbilical venous gases measured at cordocentesis. Am J Obstet Gynecol 1990;162:115-20.

9. Pourcelot L. Application cliniques de l'examen Doppler transcutane. In Velocimetrie Ultrasonore Doppler (Perroneau P. ed.), Seminaire INSERM, Paris 1974;213-40. 
10. Arduini D, Rizzo G. Normal values of pulsatility index from fetal vessels: A cross sectional study on 1556 healty fetuses. J Perinat Med 1990;18:165-72.

11. Arstrom K, Eliasson A, Hareide JH, Marsal K. Fetal blood velocity waveforms in normal pregnancies. A longitudinal study. Acta Obstet Gynecol Scand 1989;68(2):171-78.

12. Manabe A, Hata T, Kitao M. Longitudinal Doppler ultrasonographic assessment of alterations in regional vascular resistance of arteries in normal and growth-retarded fetuses. Gynecol Obstet Invest 1995;39:171-79.

13. Hecher K, Snijders R, Campbell S, Nicolaides K. Fetal venous, intracardiac, and arterial blood flow measurements in intrauterine growth retardation: Relationship with fetal blood gases. Am J Obstet Gynecol 1995;173:10-15.

14. Paulin F, Szabo I, Rigo J Jr. Doppler colour flow imaging of fetal intracerebral arteries and umbilical artery in the small for gestational age fetus. Br J Obstet Gynaecol 1995;102:174.

15. Hecher K, Campbell S, Doyle P, Harrington K, Nicolaides K. Assessment of fetal compromise by doppler ultrasound investigation of the fetal circulation arterial, intracardiac, and venous blood flow velocity studies. Circulation 1995;91:12938.

16. Aranyosi J, Major T, Fülesdi B, Zatik J. Fetal arterial redistribution indicating true umbilical cord knot. Eur J Obstet Gynecol Reprod Biol Feb 2003;10;106(2):225-26.
17. Maunu J, et al. Antenatal Doppler measurements and early brain injury in very low birth weight infants. J Pediatr 2007;150(1):51-56.

18. Konje JC, Abrams KR, Taylor DJ. Normative values of Doppler velocimetry of five major fetal arteries as determined by color power angiography. Acta Obstet Gynecol Scand 2005;84(3):23037.

19. Ertan AK, Hendrik HJ, Tanriverdi HA, Bechtold M, Schmidt W. Fetomaternal Doppler sonography nomograms. Clin Exp Obstet Gynecol 2003;30(4):211-16.

20. Parra-Cordero M, Lees C, Missfelder-Lobos H, Seed P, Harris C. Fetal arterial and venous Doppler pulsatility index and time averaged velocity ranges. Prenat Diagn Dec 2007;27(13):125157.

21. Jugović D, Tumbri J, Medić M, Jukić MK, Kurjak A, Arbeille P, Salihagić-Kadić A. New Doppler index for prediction of perinatal brain damage in growth-restricted and hypoxic fetuses. Ultrasound Obstet Gynecol Sep 2007;30(3):303-11.

22. Tchirikov M, Strohner M, Förster D, Hüneke B. A combination of umbilical artery PI and normalized blood flow volume in the umbilical vein: Venous-arterial index for the prediction of fetal outcome. Eur J Obstet Gynecol Reprod Biol Feb 2009;142(2):129-33.

23. Nishihara R, Nakai Y, Tachibana D, Yamamasu S, Iwanaga N, et al. Effects of different sampling points on evaluation of fetal descending aortic flow. Osaka City Med J Jun 2006;52(1):3945 . 